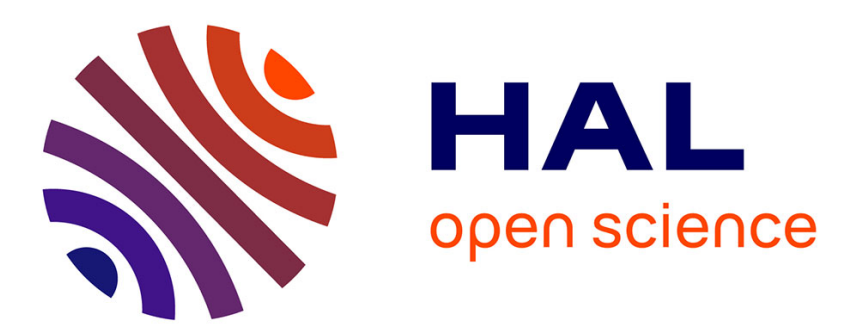

\title{
Rôle du poteyage et de la température initiale du moule sur les sollicitations thermomécaniques des moules permanents de fonderie
}

\author{
Gilles Dour, A. Broucaret, Alexis Oudin, Abderrahim Michrafy, Farhad \\ Rezai-Aria
}

\section{To cite this version:}

Gilles Dour, A. Broucaret, Alexis Oudin, Abderrahim Michrafy, Farhad Rezai-Aria. Rôle du poteyage et de la température initiale du moule sur les sollicitations thermomécaniques des moules permanents de fonderie. Matériaux \& Techniques, 2000, ${ }^{\circ}$ Hors série, p.45-50. 10.1051/mattech/200088120045s . hal-01847830

\section{HAL Id: hal-01847830 \\ https://hal.science/hal-01847830}

Submitted on 6 Nov 2019

HAL is a multi-disciplinary open access archive for the deposit and dissemination of scientific research documents, whether they are published or not. The documents may come from teaching and research institutions in France or abroad, or from public or private research centers.
L'archive ouverte pluridisciplinaire HAL, est destinée au dépôt et à la diffusion de documents scientifiques de niveau recherche, publiés ou non, émanant des établissements d'enseignement et de recherche français ou étrangers, des laboratoires publics ou privés. 


\title{
Rôle du poteyage et de la température initiale du moule sur les sollicitations thermomécaniques des moules permanents de fonderie
}

\author{
G. Dour, S. Broucaret, A. Oudin, A. Michrafy et F. Rézaï-Aria \\ Ecole des Mines d'Albi-Carmaux, Albi
}

\section{EFFECT OF COATING AND INITIAL DIE TEMPERATURE \\ ON THERMAL STRESSES INTO DIE CASTING DIES}

ABSTRACT: In the casting industry metal moulds or dies are used more and more. The reason is that they allow a fast cooling rate of the solidifing part, hence allowing higher productivity, finer microstructure and higher mechanical properties. In most cases the die is made out of steel and reacts with the liquid cast metal. The usual solution is to cover the moulding surface with a coating or spray. Depending on the casting technology, the coating is sprayed every cycle or every 8 tolo hours of production. A second but nonetheless important effect of the coating is its thermal effect. The coating acts as a thermal barrier and protects the die against thermal shocks. The topic of the present paper is to assess this function of the coating.

During a casting cycle, the coated die and the molten metal are briefly in contact during the very first moments and then an air gap may form and separate them apart. During the first stage, an intense heat is transferred from the melt to the die. Heat flux densities from $0.5 \mathrm{MW} / \mathrm{m}^{2}$ up to $10 \mathrm{MW} / \mathrm{m}$ have been reported in literature. The intense heat transfer generates high temperature heterogeneity into the die. The corresponding dilatation heterogeneity is responsible for internal stresses into the die, so called thermal stresses. They are usually compressive stresses on the hot surface. It will be shown in this paper that the moulding surface of the die suffers the most stress. The stresses can be high enough to cause yielding of the steel at high temperature. Because the steels in use for dies have a high yield stress at high temperature the plastic deformation remains small. However it is a cyclic plasticity because the same phenomenon occurs at every casting cycle. We believe that this plasticity in warm conditions is responsible for residual tensile stresses in cold conditions (i.e. nearly isothermal conditions). This phenomenon is rather classical in most thermal stresses problems [1, 2]. At the life-time scale of the die, the moulding surface is cyclically stressed in traction at low temperatures and compression at high temperatures leading to a fatal cracking.

In a first approach we suggest measuring temperatures within the die during a casting cycle. From this measurement it is possible to estimate the thermal stresses, assuming that the stresses remain below or at least close to the yield stress of the die materials. This assumption is usually fair. Indeed, if the plastic deformation were large during every cycle, the die would never last much longer than a few thousands cycles. If this ever occurred, it would not be a great advantage for the casting factory nor its client. Other materials should be sought as a first priority. From the estimation of thermal stresses, it would be delicate to foresee the mechanical behaviour of the materials of the die in fatigue condition. Some materials tend to harden (copper alloys, fcc materials) while others tend to soften (heat treated martensite steels [4])[5]. Instead of trying to guess the behaviour, the method that we suggest is to perform a thermomechanical fatigue test (TMF). This TMF test consists of applying measured temperature and evaluated strain/stresses history to a mechanical testing sample [6]. The most relevant temperature and stress history is, of course, the one corresponding to the moulding surface of the die. This test will provide information on the materials behaviour and some relevant data about the lifetime of the die.

Conférence présentée au colloque "Moules et outils 2000 - Interactions avec le produit mis en auvre et solutions » les 17 et 18 mai 2000 à SaintÉtienne. lors des $39^{\circ}$ journées du Cercle d'Études des Métaux, qui l'a organisé en collaboration avec la section Sud-Est de la SF2M et l'Agence RhôneAlpes pour la maîtrise des matériaux.

This paper provides an example of this method. The thermal data was obtained from a gravity casting experiment [3] that is described in the first part. The second part deals with the evaluation of the thermal stresses and the third part shows some results from the TMF testing. Throughout the paper the influence of the coating nature and of the die initial temperature is examined. KEY WORDS: Thermal fatigue, heat transfer, thermal stresses, thermo-mechanical fatigue, surface coating, spray. 
RÉsumÉ: Cet article traite de la fatigue thermique subie par les outillages de mise en forme. Pour appréhender le problème d'endommagement des outillages, il est nécessaire de bien connaître les conditions de transfert de chaleur et d'évaluer les contraintes thermo-mécaniques subies par l'outillage. La prévision de la durée de vie et l'évolution de la plasticité cyclique qui précède la fissuration est difficile car certains matériaux ont tendance à s'écrouir alors que d'autres ont tendance à s'adoucir. Nous proposons le recours à des expériences de fatigue thermo-mécanique (TMF) pour appréhender cette prévision. Par un exemple emblématique, nous montrons comment il est possible d'aborder le problème. Le cas considéré est la fonderie gravité en coquille d'acier. Nous mettons en évidence l“influence de paramètres process sur le transfert de chaleur et sur les contraintes thermo-mécaniques subies par le moule pendant un cycle de coulée. Les paramètres retenus sont la température initiale du moule et la nature du poteyage. Des essais de fatigue thermo-mécanique ont été menés et les résultats sur 1000 cycles sont exposés.

MOTS CLÉS: Fatigue thermique, transfert thermique, contraintes ther miques, fatigue thermo-mécanique, poteyage.

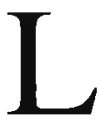

es moules de fonderie, comme tous les outillages de mise en forme, subissent des chocs thermiques répétés.

La matière à mettre en forme est en effet portée à chaud pour la mettre en forme contre un outil froid. Ce type de chocs subis par l'outil est qualifié de choc chaud, par opposition aux chocs froids que subit par exemple une pièce pendant une trempe. Pendant un choc chaud, la face chauffée est soumise à des contraintes thermiques en compression et l'intérieur en traction. Le choc chaud pendant la mise en forme d'une pièce n'est généralement pas suffisamment intense pour engendrer la ruine immédiate de l'outil. Cependant, la répétition de ces chocs conduit irrémédiablement les moules à s'endommager. En effet, pendant le choc chaud, la température s'élève et la limite élastique diminue. Par conséquent, si la sollicitation thermomécanique dépasse la limite élastique. le matériau peut se déformer plastiquement. L'effet de cette plasticité à chaud se fait sentir à froid (conditions quasi-isotherme) par le développement de contraintes résiduelles en traction sur la face chauffée. Cette situation est classique dans les problèmes de thermomécanique [1,2]. La répétition des chocs thermiques chauds engendre une plasticité cyclique. Cette plasticité, aussi petite soit-elle, est à l'origine de l'endommagement par fissuration des outillages. Comme nous le décrivons dans cet article, la température et la contrainte sur la face moulante des moules sont les plus élevées (en valeur absolue). Ceci, combiné à l'effet de l'environnement, explique que la fissuration est observée surtout en surface. Parce que l'état de contrainte thermo-mécanique est bi-axial, un réseau bi-axial de fissures, autrement appelé faiiençage, se développe, comme en fonderie sous pression, en forge, pour la mise en forme du verre, etc.

Dans une expérience reprise dans cet article, mais décrite plus complètement dans [3], nous mettons en évidence l'influence de paramètres process sur les contraintes thermo-mécaniques subies par le moule pendant un cycle de coulée. Les paramètres retenus sont la température initiale du moule et la nature du poteyage. En pratique, par ces deux paramètres nous jouons sur la densité de flux ( $h . \Delta T$ comme on l'écrit généralement) transmise au moule dans les premiers instants de la coulée (avant que se forme une lame d'air entre le moule et la pièce). Il est possible d'établir une relation entre densité de flux imposée et contrainte thermo-mécanique. Retenons pour l'instant que plus cette densité de flux est grande, plus les contraintes subies sont grandes et plus les températures tendent à être élevées. Tout ceci concourt dans le sens d'une plus grande déformation plastique cyclique. Pour appréhender le problème d'endommagement des outillages il est donc nécessaire de bien connaître les conditions de transfert de chaleur et d'évaluer les contraintes thermo-mécaniques. La prévision de la durée de vie et l'évolution de la plasticité cyclique qui précède la fissuration est difficile car certains matériaux ont tendance à s'écrouir (alliages de cuivre, matériaux fcc) alors que d'autres ont tendance à s'adoucir (aciers martensitiques revenus [4]) [5]. Nous proposons le recours à des expériences de fatigue thermo-mécanique (TMF) pour appréhender cette prévision. Cet essai consiste à soumettre une éprouvette de fatigue à un cycle thermique et mécanique imposé [6]. Les histoires imposées sont calquées sur celles mesurées et évaluées respectivement pour la température et pour la déformation, à proximité de la surface moulante.

Nous proposons donc d'aborder les problèmes de fatigue thermique par une approche globale en trois étapes : mesure du champ de température dans l'outillage, évaluation des contraintes thermo-mécaniques, essai TMF. Nous fournissons ici un exemple d'application de cette méthode. Les données thermiques sont issues d'une expérience menée dans les conditions de la coulée gravité [3] et décrites dans le premier paragraphe. Les contraintes de choc thermique subies par un moule de fonderie, hors zone de singularité géométrique, sont abordées dans le second paragraphe. Enfin, l'essai TMF a été employé pour simuler l'histoire thermo-mécanique telle qu'évaluée pour un élément proche de la surface moulante et les résultats sont reportés dans le troisième paragraphe.

\section{Mesures thermiques et estimation du champ de température d'un moule de fonderie gravité}

Nous avons équipé un moule de fonderie gravité d'un capteur de température de type fluxmètre $1 \mathrm{D}$. Celui-ci est conçu comme un insert de $10 \mathrm{~mm}$ de diamètre environ et aussi épais que le moule $(40 \mathrm{~mm})$. Il peut être remplacé par un insert de mêmes dimensions après usage. Quinze thermocouples ont été implantés par brasure dans l'insert. La disposition est détaillée dans la figure 1 . Le matériau du moule et du capteur est un acier XC 35 traité à $42 \mathrm{HRc}$.

Le moule est de géométrie simple ( $c f$. fig. 1), car il permet de couler latéralement une pièce de géométrie parallélépipédique. Le capteur est placé au milieu du parallélépipède. Le capteur est tronc-conique et son implantation dans le moule se fait par l'extérieur. Son maintien en position est assuré par la table de fixation du moule sur le mécanisme d'ouverture-fermeture du moule (en face arrière).

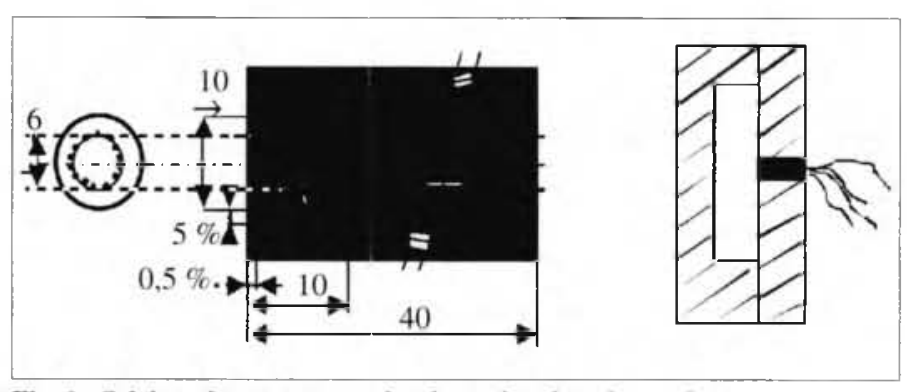

Fig. 1 - Schéma du capteur son implantation dans le moule.

Fig. I-Schematic view of the heat flux gauge and its position in the die. 
Dans un premier temps, le capteur a été vérifié au moyen d'un test spécifique. Nous réalisons un choc froid avec un glaçon déposé contre le moule préalablement chauffé à $180-220^{\circ} \mathrm{C}$. Nous mesurons les températures aux 15 points de mesures. Par l'application d'une méthode inverse [7] et par simple différence de température, nous déterminons la densité de flux de chaleur délivrée au glaçon pour fondre. Intégrée dans le temps, cette densité de flux doit être équivalente à l'énergie surfacique consommée par le glaçon pour fondre. La corrélation est bonne [3] pour les ordres de grandeur. L'instrumentation devra être améliorée pour affiner la corrélation.

Nous avons réalisé des coulées d'alliage d'aluminium (AS 7G 06) à $750^{\circ} \mathrm{C}$ dans le moule revêtu soit d'un poteyage à base de graphite collö̈dal, soit à base de réfractaire isolant (ci-dessous appelé alumine). Les deux poteyages ont été appliqués sans viser une épaisseur particulière, contrairement à [8]. Le poteyage graphite a une épaisseur de 10-20 $\mu \mathrm{m}$ et le poteyage alumine 150-200 $\mu \mathrm{m}$. Le moule est porté à chaque fois à une température initiale de 200, 240 puis $30{ }^{\circ} \mathrm{C}$. Chaque essai a été doublé par températures décroissantes 300,240 et enfin $200^{\circ} \mathrm{C}$. Chaque poteyage a été conservé pendant toute la durée d'une série, soit 6 coulées successives par poteyage.

La figure 2 présente les courbes température en fonction du temps pour chaque thermocouple. La température initiale du moule était de $240^{\circ} \mathrm{C}$ pour ces deux essais. A gauche se trouvent les courbes obtenues avec le poteyage graphite et à droite avec le poteyage alumine.

La figure 3 représente les densités de flux estimées par différence de température entre deux thermocouples, à l'équivalent de $1 \mathrm{~mm}, 4,75 \mathrm{~mm}$ et $8,5 \mathrm{~mm}$ de la surface moulante respectivement.
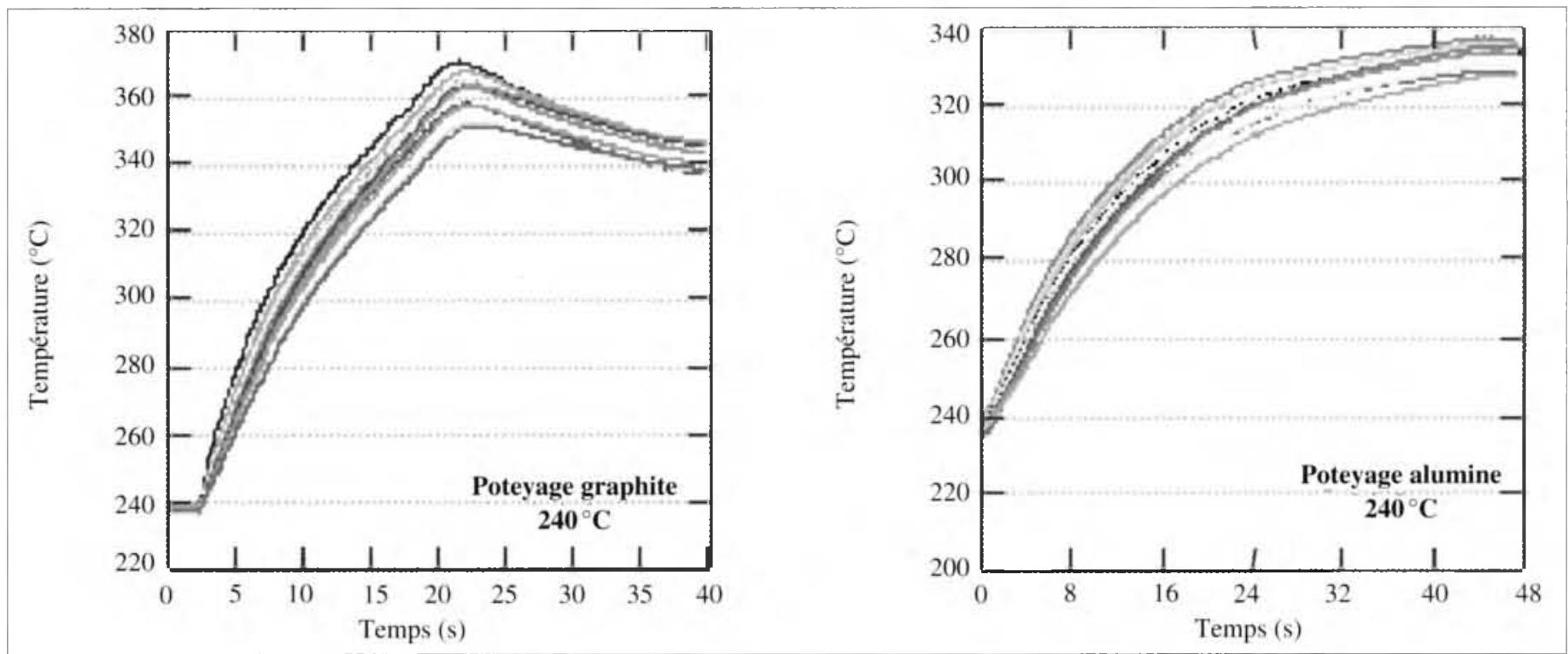

Fig. 2 - Courbes typiques températures-temps enregistrées par thermocouples pendant les coulées avec poteyage graphite (à g.) et poteyage alumine (à dr.).

Fig. 2 - Typical curves temperature vs. time on each thermocouple for (left) a graphite based coating and (right) an alumina based coating.
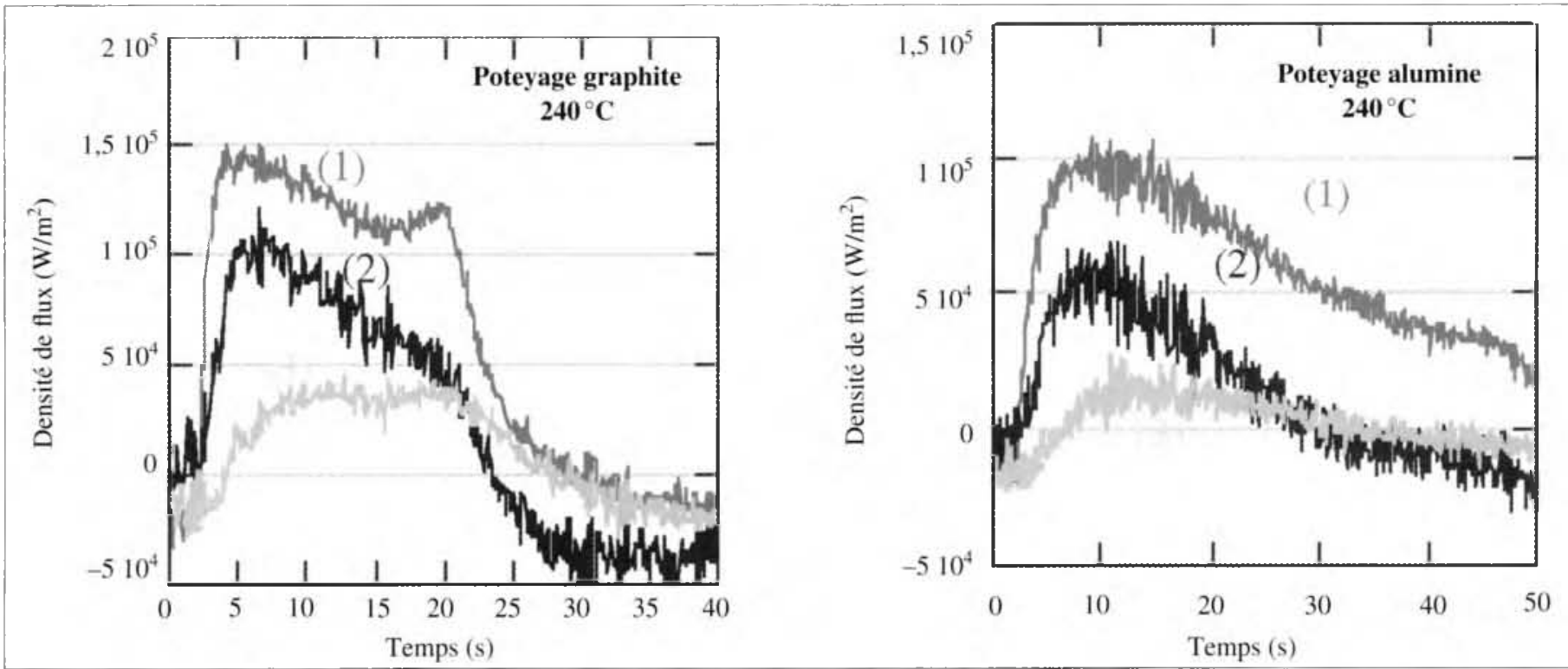

Fig. 3 - Courbes densité de flux-temps pour différentes positions 1, 2 et 3 et pour deux coulées avec poteyage graphite (gauche) et alumine (droite). (1) flux estimé à partir des thermocouples 1 et 3, (2) flux estimé à partir des thermocouples 9 et 10, (3) flux estimé à partir des thermocouples 13 et 14.

Fig. 3 - Curves Heat Flux Density vs. Time at different positions 1, 2 and 3 with the two coatings and the same initial temperature (see paragraph 3), (1) being the fux derived from thermocouples 1 and 3, (2) being the flux derived from thermocouples 9 and 10, (3) being the flux derived from thermocouples 13 and 14 . 
Nous avons résumé l'impact des paramètres process que sont la température initiale du moule et le poteyage utilisé sur les figures 4 et 5 .

La figure 4 présente l'effet des paramètres sur la vitesse de montée de la température à $0,5 \mathrm{~mm}$ de la surface moulante et à l'instant initial de sa montée. Il est clair que le poteyage a une influence marquée sur la vitesse de montée de température. La température initiale a un effet, mais moindre. Notons que les résultats sont très reproductibles pour le poteyage alumine, contrairement au poteyage graphite.

La figure 5 présente les densités de flux maximale relevées à $1 \mathrm{~mm}$ de la face moulante en fonction des paramètres process (à droite). Comme pour les vitesses de montée de température, le poteyage apparaît comme le paramètre le plus influent.

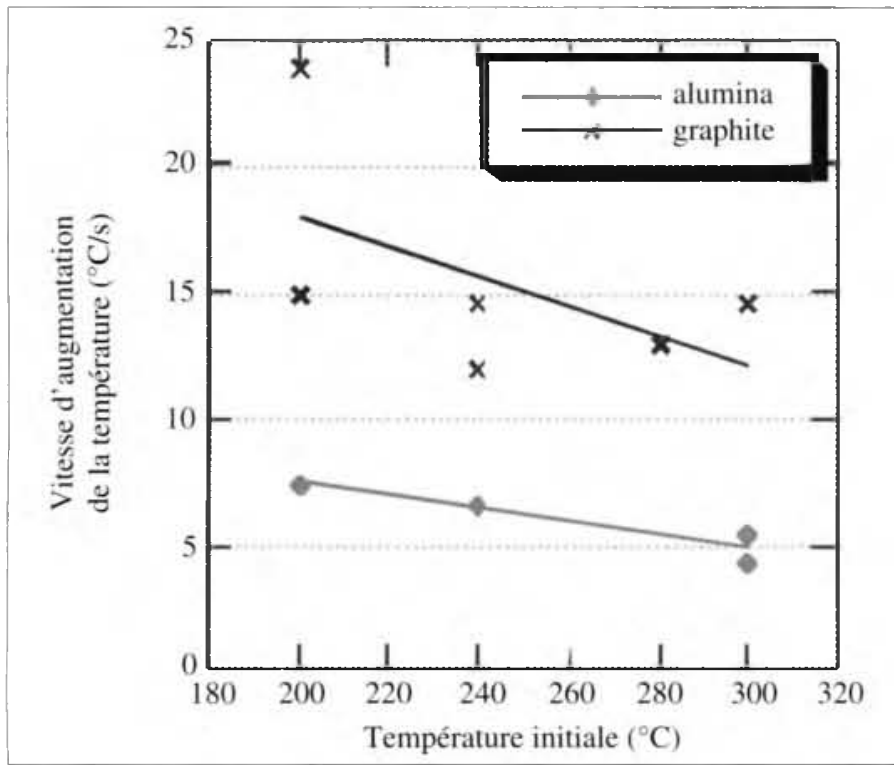

Fig. 4 - Influence du poteyage et de la température initiale sur la vitesse de montée de la température à $0.5 \mathrm{~mm}$ de la surface moulante.

Fig. 4 - Influence of the coating and of the initial temperature on the temperature increase rate at the very first instant of the casting.
Notons encore une fois une différence notable sur la reproductibilité des résultats entre type de poteyage.

La faible reproductibilité des résultats obtenus avec le poteyage graphite mérite une réflexion. L'observation des courbes de la figure 5 (gauche) pour le graphite nous laisse penser que le poteyage graphite neuf $\left(240^{\circ} \mathrm{C}-1\right)$ a une réaction avec l'alliage coulé différente que le même poteyage après service $\left(240^{\circ} \mathrm{C}-2\right)$. En effet, la courbe de flux du poteyage neuf présente une décroissance de flux suivi d'une remontée. Cela ressemble beaucoup à un phénomène de recalescence, liée à une surfusion forte du bain.

\section{Évaluation des contraintes thermo-mécaniques dans l'outillage de géométrie simple}

Connaissant le champ de température dans le moule de géométrie simple (ici une plaque), il est possible d'évaluer les contraintes dans le moule. Nous supposons que le moule est autobridé, c'est-à-dire qu'il est libre de se déformer sous la sollicitation thermo-mécanique. Nous supposons également que les niveaux de contraintes atteints sont suffisamment faibles pour que le matériau se comporte élastiquement. Dans ce cas, un développement de thermo-élasticité montre que le champ de contrainte est bi-axial, avec les deux contraintes principales tangentielles à la surface du moule [9]. Celles-ci sont égales et dépendent de l'épaisseur $x$ et du temps $t$, suivant les expressions (1), (2) et (3).

La figure 6 (gauche) montre le profil de ces contraintes dans l'épaisseur du moule, au moment où la contrainte compressive en face moulante est la plus forte. Cela correspond à des instants distincts, selon les paramètres process. Pour le cas particulier du moule porté initialement à $240^{\circ} \mathrm{C}$. l'instant de contraintes maximales est de $2,1 \mathrm{~s}$ pour le poteyage graphite et de $4,5 \mathrm{~s}$ pour l'alumine. En particulier aussi, les températures atteintes en face moulante ne sont pas à leur maximum. Ainsi pour le poteyage graphite, la température reportée en figure 8 est de $275^{\circ} \mathrm{C}$, contre $370{ }^{\circ} \mathrm{C}$ de température maximale pendant la coulée.

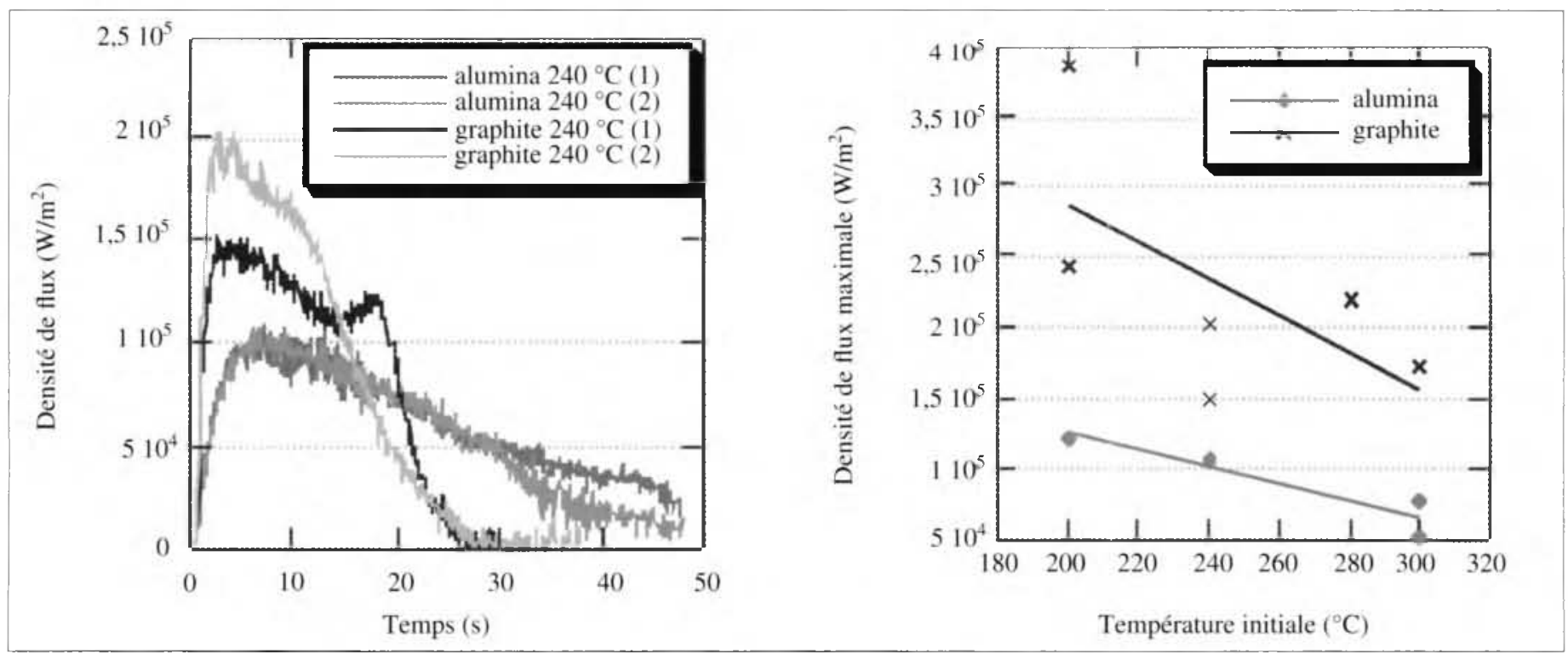

Fig. 5 - Influence de l'ordre des coulées sur l'évolution des flux en position 1 (gauche). Influence du poteyage et de la température initiale sur le pic de flux (droite).

Fig. 5 - Influence of the testing procedure on the heat fux at $0,5 \mathrm{~mm}$ inside the die (left). Influence of both the coating and the initial temperature on the maximum heat flux estimated on the moulding surface (right). 


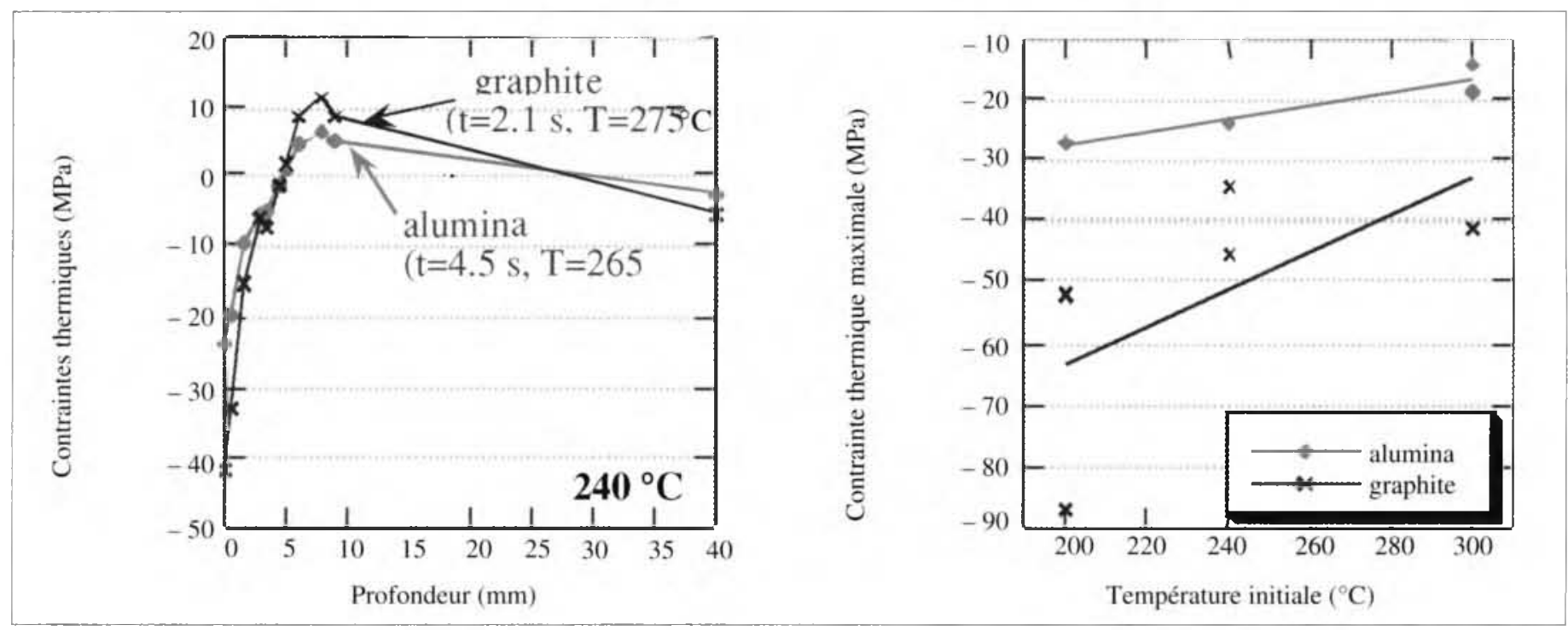

Fig. 6 - Profil des contraintes dans l'épaisseur du moule au moment où les contraintes sont les plus importantes (gauche). Influence du poteyage et de la température initiale sur les contraintes compressives maximales sur la face moulante (droite).

Fig. 6 -Maximum compressive thermal stresses within the die for the two coatings and the same initial temperature $240{ }^{\circ} \mathrm{C}$ (left). Maximum compressive thermal stresses at the moulding surface of the die vs. initial temperature for the two coatings (right).

La figure 6 (droite) résume les contraintes compressives maximales observées en fonction des paramètres process. Encore une fois, on observe que le poteyage a une influence plus marquée que la température initiale. Notons également que les résultats sont moins reproductibles avec le poteyage graphite qu'avec le poteyage alumine. L'effet de la réactivité du poteyage pourrait être utilisé comme protection mécanique du moule de fonderie. En effet, les contraintes associées au poteyage graphite neuf à $200{ }^{\circ} \mathrm{C}$ sont de $45 \mathrm{MPa}$ contre $90 \mathrm{MPa}$ pour le poteyage usagé.

$$
\begin{gathered}
\sigma(x, t)=\frac{1}{1-v}\left\{\alpha E\left[-\mathrm{T}(x, t)+\frac{1}{2 h} N_{t}+\frac{3(x+h)}{2 h^{3}} \Pi_{t}\right]\right\} \\
N_{t}=\int_{0}^{2 h} T(x, t) d x \\
\Pi_{t}=\int_{0}^{2 h}(x+h) \mathrm{T}(x, t) d x
\end{gathered}
$$

\section{Essai de fatigue thermo-mécanique}

Nous avons repris les courbes température et déformation mécanique, en fonction du temps, subies par le matériau situé sur la face moulante du moule pour l'essai n ${ }^{\circ} 2$ à $200^{\circ} \mathrm{C}$ de température initiale et un poteyage graphite. Comme prévu par les estimations thermo-élastiques, la contrainte compressive la plus grande n'est subie qu'à température de $270{ }^{\circ} \mathrm{C}$ environ. La contrainte est alors de $-100 \mathrm{MPa}$, ce qui correspond à une déformation mécanique de $-0,035 \%$. Nous avons reproduit ce cycle thermo-mécanique sur une machine de fatigue, tel que présenté sur la figure 7.

La figure 8 montre les cycles d'hystéresis subis par une éprouvette en XC35 traitée à $42 \mathrm{HRc}$ en début de cyclage ( 10 cycles) et au bout d'un grand nombre de cycles (900 cycles). Nous constatons que l'hypothèse de déformation élastique est vérifiée de façon satisfaisante. En outre, il y a peu d'évolution de la

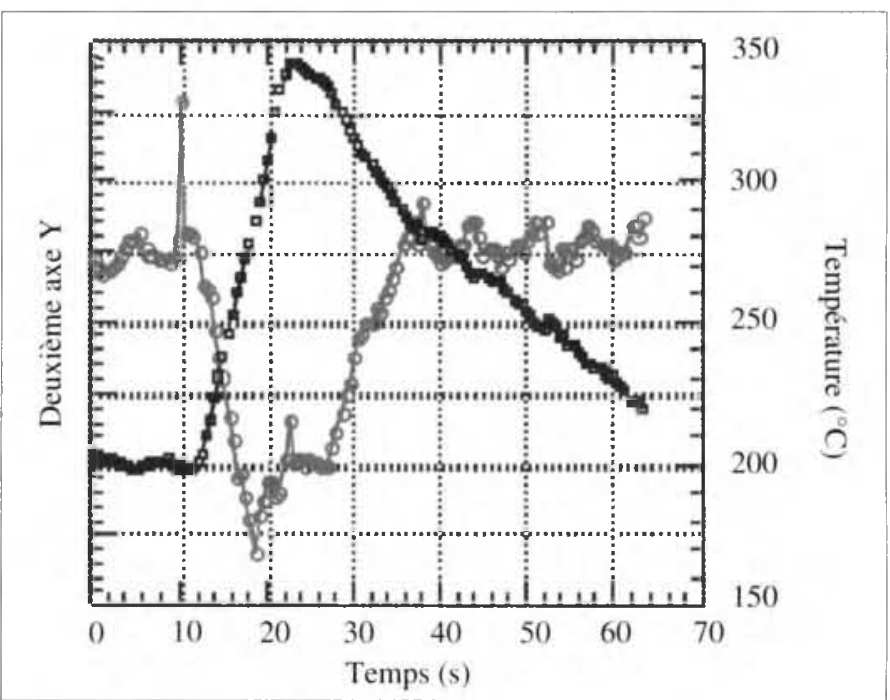

Fig. 7 - Cycle de fatigue thermo-mécanique subi par un élément de moule placé près de la face moulante.

Fig. 7 - Thermo-mechanical fatigue cycle as evaluated and tested for the volume element considered in the vicinity of the moulding surface.

boucle d'hystérésis contrainte-déformation sur 900 cycles. Le matériau ne subit donc pas d'évolution (écrouissage ou adoucissement cyclique) significative sur ce nombre de cycle. Pour constater une telle évolution, il faudrait faire un test jusqu'à plusieurs 100000 cycles, voire plus puisque les déformations mécaniques sont très faibles comparées à celle reportées en [6]. N'oublions pas que les chocs thermiques de la fonderie gravité sont faibles (flux maximum de $0,5 \mathrm{MW} / \mathrm{m}^{2}$ ) alors que d'autres procédés peuvent présenter des chocs bien plus importants (on reporte des flux de 5 à $20 \mathrm{MW} / \mathrm{m}^{2}$ en fonderie d'acier contre une paroi froide - réunions du groupement d'étude des transferts thermiques à l'interface GETTI). Enfin cette faiblesse des chocs thermiques en fonderie gravité justifie amplement l'utilisation d'un acier « ordinaire » plus conducteur de la chaleur $\left(43-50 \mathrm{~W} / \mathrm{m}^{2} \mathrm{~K}\right)$ qu'un acier « spécial » très chargé en élément d'alliage et de ce fait moins bon conducteur de la chaleur $\left(25\right.$ à $\left.30 \mathrm{~W} / \mathrm{m}^{2} \mathrm{~K}\right)$. 


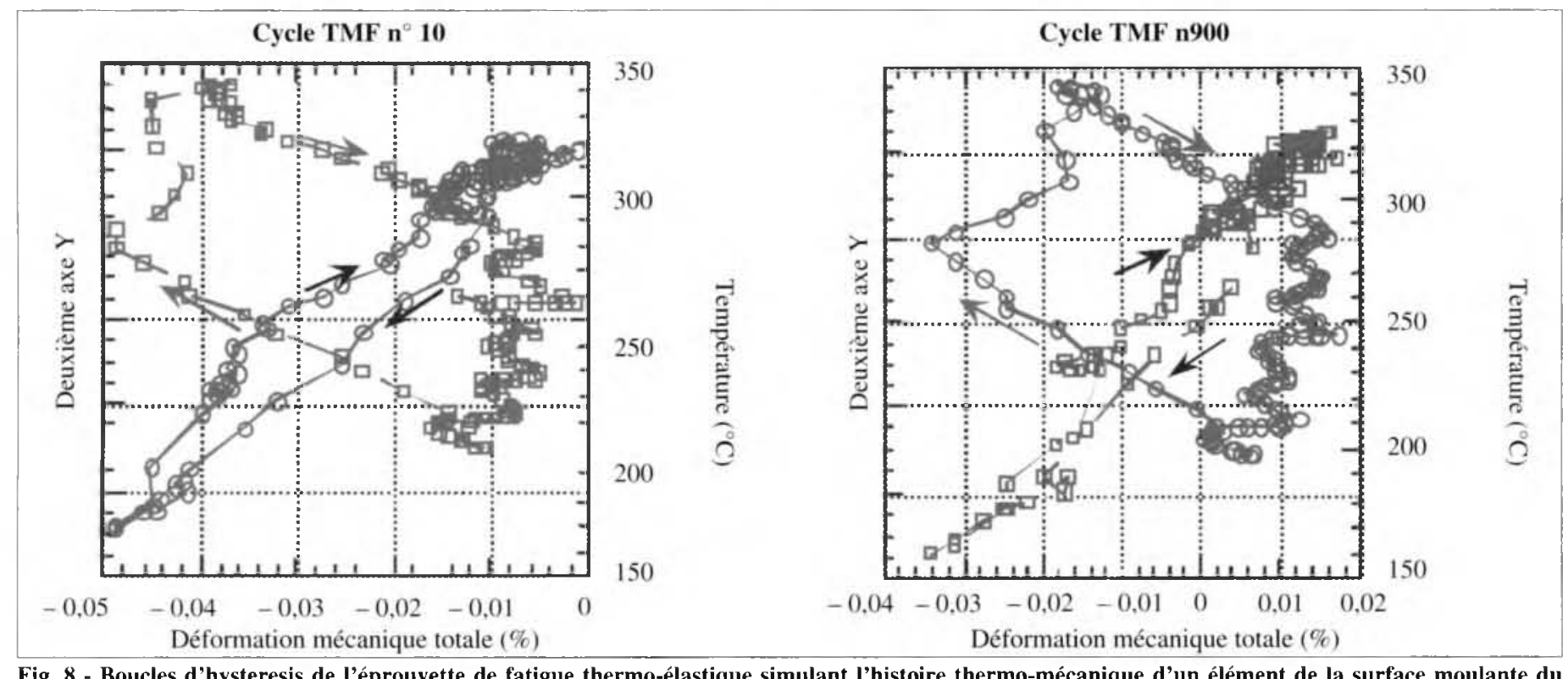

Fig. 8 - Boucles d'hysteresis de l'éprouvette de fatigue thermo-élastique simulant l'histoire thermo-mécanique d'un élément de la surface moulante du moule.

Fig. 8 - Hysteresis loops for the thermo-mechanical test representing the thermo-mechanical history of a volume elcment next to the moulding surface.

\section{Conclusion}

Nous avons développé une méthodologie 1D d'analyse thermique et mécanique dans un moule de géométrie simple. Cette méthode fournit des informations sur la sollicitation thermo-mécanique subie par le matériau de moule à différentes épaisseurs et en particulier sur la face moulante qui s'endommage en premier. Il est alors possible de reproduire ce cyclage thermique et mécanique sur une éprouvette de fatigue dite thermo-mécanique. Il est montré que les deux paramètres process choisis (température initiale du moule et poteyage) ont une influence sur le transfert de chaleur entre pièce et moule, et sur les contraintes thermo-mécaniques. Parmi les deux paramètres process, la nature du poteyage (à travers ses propriétés intrinsèques mais aussi son mouillage de la surface) est le plus influent. En particulier, le vieillissement du poteyage graphite semblerait jouer un rôle important sur la transmission de la chaleur et par conséquent sur les contraintes thermo-mécaniques.

Les perspectives de ce travail sont les suivantes:

- Cette méthodologie a été appliquée à des outillages de fonderie subissant un faible chargement thermo-mécanique, du fait de la protection thermique du moule (poteyage). Il est possible cependant d'appliquer la même méthode à des outils subissant des sollicitations plus violentes. Alors, la durée de vie de l'éprouvette de fatigue thermo-mécanique servirait à fournir une estimation de la durée de vie d'un moule soumis à un cycle thermo-mécanique préalablement évalué partir de mesures de flux. Il est cependant bien évident que chaque zone d'un moule peut subir des sollicitations thermiques, et donc mécaniques, différentes. Alors il convient de mener l'étude sur les zones les plus sensibles au faiençage d'un moule donné.

- L'évaluation de l'état de contrainte du moule a été réalisée en supposant que le matériau se comporte élastiquement. Si cela s'est avéré correct dans notre cas, il n'en sera pas de même pour des sollicitations thermiques plus violentes. Il est cependant possible d'utiliser une expression analytique du même type que celle utilisée [10] pour faire une évaluation plus précise. Il convient alors d'utiliser des tests spécifiques pour identifier la vitesse de déformation plastique à chaque incrément de temps et l'intégrer dans l'expression en question [11].

\section{RÉFÉRENCES}

[1] L.J. EBERT, The role of residual stresses in the mechanical performance of case carburised steels, Met. Trans, vol 9A, nov 78, 1537.

[2] G. Dour, Tirage semi-continu de silicium photovoltaïque multicristallin en creuset froid inductif. PhD report, Chap III. INP Grenoble, 1996.

[3] S. Broucaret, A. Michrafy, B. Ladevie, G. Dour, Heat Transfer in a Gravity Gasting Die - Influence of the Process Parameters - SIM 2000 - Aachen August 2000 - in press.

[4] D. Delagnes, Comportement et tenue en fatigue isotherme d'aciers à outil Z 38 CDV 5 autour de la transition fatigue oligocyclique-endurance - PhD report, École des Mines Paris, 1998.

[5] R.K. HAM. The metallurgy of transition-life fatigue Monograph and Report Series - Metals and Metallurgy Trust, $n^{\circ} 32$, pp. 55-79, 1967.

[6] A. Oudin, L. Penazzi, F. Rézai-Aria, Prédiction de la durée de vie en fatigue thermique des outillages de mise en forme à chaud à l'aide d'essais de fatigue thermo-mécanique $-39^{\circ}$ Journée du
Cercle d’Étude des Métaux, 17-18 mai 2000. Matériaux \& Techniques, $\mathrm{n}^{\circ}$ hors série, déc. 2000 (présent recueil) p. 00-00.

[7] O. Fudym, C. Carrere-Gée, D. Lecomte, B. Ladevie, Heat Flux estimation in thin-layer drying - Inverse Problems in Engineering: Theory and Practice $-3^{\text {rd }}$ Int. Conf. On Inverse. Problems in Engineering - June 13-18, 1999, Port Ludlow, WA, USA.

[8] G. Sciama, J.F. Ribol, Solidification de barreaux en AISi7Mg coulés contre refroidisseurs, Fondeur et Fonderie d'Aujourd'hui, n ${ }^{\circ}$ 169, pp. 23-61 Nov 1997.

[9] B.A. BOLEY, J.H. WeINER, Theory of Thermal Stresses, Krieger Ed., 1968.

[10] S. Suresh, A.E. Giannakopoulos, M. Olsson, Sharp interfees versus Functionally Graded Metal-Ceramic layers : Geometrical optimization for mechanical response $-3^{\text {rd }}$ Int. Symp. On Structural and Functional Gradient Materials - 1994.

[11] G. Dour, F. Durand, Y. BRÉCHET, Relaxation of thermal stresses by dislocation flow and multiplication in the continuous casting of silicon-Modeling Simul Mater. Sci. Eng., 5 (1997) 275-288. 